\title{
Diagnostic accuracy and feasibility of a rapid SARS-CoV-2 antigen test in general practice - a prospective multicenter validation and implementation study
}

Jörn Rohde ( $\square$ rohde_j@ukw.de)

Julius-Maximilians-Universität Wuerzburg

Wolfgang Himmel

University Medical Centre Goettingen

Clemens Hofinger

Julius-Maximilians-Universität Wuerzburg

Thiên-Trí Lâm

Julius-Maximilians University of Wuerzburg

Hanna Schrader

Julius-Maximilians-Universität Wuerzburg

Julia Wallstabe

Julius-Maximilians University of Wuerzburg

Oliver Kurzai

Julius-Maximilians University of Wuerzburg

Ildikó Gágyor

Julius-Maximilians-Universität Wuerzburg

\section{Research Article}

Keywords: COVID-19 Testing, general practice, sensitivity and specificity, attitude of health personnel, feasibility study

Posted Date: February 17th, 2022

DOI: https://doi.org/10.21203/rs.3.rs-1336366/v1

License: (a) (1) This work is licensed under a Creative Commons Attribution 4.0 International License. Read Full License 


\section{Abstract \\ Background}

PCR testing is considered the gold standard for SARS-CoV-2 diagnosis but its results are earliest available hours to days after testing. Rapid antigen tests represent a diagnostic tool enabling testing at the point of care. To date, most rapid antigen tests were validated by the manufacturer or in controlled laboratory settings only. External validation at the point of care, particularly in general practice where the test is frequently used, is needed. Furthermore, it is unclear how well point of care tests are accepted by the practice staff.

\section{Methods}

In this prospective multicenter validation study in primary care, general practitioners included adult individuals presenting with symptoms suggesting COVID-19. Each patient was tested first with a nasopharyngeal swab for the point of care test and then with a second swab for PCR testing. Using the RT-PCR result as a reference, we calculated specificity, sensitivity, positive predictive value and negative predictive value, with their $95 \%$ confidence intervals. General practitioners and medical assistants completed a survey to assess feasibility and usefulness of the point of care tests.

\section{Results}

In 40 practices in Würzburg, Germany, 1518 patients were recruited between 12/2020 and 06/2021. The point of care test achieved a sensitivity of $78.3 \%$ and a specificity of $99.5 \%$ compared to RT-PCR. With a prevalence of $9.5 \%$, the positive predictive value was $93.9 \%$ and the negative predictive value was $97.8 \%$. General practitioners rated the point of care test as a helpful tool to support diagnostics in patients with signs and symptoms suggestive for infection, particularly in situations where decision on further care is needed at short notice.

\section{Conclusion}

The point of care test used in this study showed a sensitivity below the manufacturer's specification (Sensitivity $96.25 \%$ ) in the practice but high values for specificity and high positive predictive value and negative predictive value. Although widely accepted in the practice, measures for further patient management require a sensitive interpretation of the point of care test results.

\section{Introduction}

Reverse transcription polymerase chain reaction (RT-PCR) tests are considered the gold standard for the diagnosis of SARS-CoV-2 infections $(1,2)$. However, they have some limitations e.g. a long turnaround time reaching up to 24 hours or more, high demands regarding infrastructure, and relatively high costs (3-5). Suitable alternatives particularly for the use in primary care could be rapid antigen tests as already demonstrated, e.g. for the diagnosis of streptococcal tonsillitis or influenza (6-8). These tests should be characterized by rapid and cost-effective results, user-friendly handling, and low infrastructural requirements (9). The pandemic has increased the overall workload of general practitioners (GPs) (10). In this context, it is important to investigate how well a rapid test can actually get integrated into the already stressful workday. 
Rapid tests must have a sensitivity of at least $80 \%$ and a specificity of at least $97 \%$ for approval in Germany by the Federal Institute for Drugs and Medical Devices (BfArM) (11). Most of them were validated by the manufacturer only. However, external validation at the point of care, where the test is frequently used, is needed. GPs play a key role in the pandemic as they are usually the first contact for patients. About $34 \%$ of corona cases identified in the first half of 2020 were treated by the GPs (12). In addition to patient care involving diagnosis, treatment and patient education, general practice is determined to be a place to go for testing $(13,14)$.

The validation studies of the manufacturers often include only small study samples with a very high prevalence. These study populations are not comparable to real world health care. While there are independent validations performed under controlled laboratory conditions that already provide valuable information, there are only few studies conducted in primary care settings $(15,16)$. A study in an urban health center in Madrid, Spain found a $100 \%$ specificity of the Abbott's Panbio ${ }^{\mathrm{TM}}$ COVID-19 Ag Rapid Test Device and 72\% sensitivity, but included only a sample of 103 patients and was conducted in a district with high incidence of Covid-19 infections (17). A study in a test station in Oslo, Norway, was conducted in a low to medium prevalence setting and found sensitivities between 55 and $79 \%$ for the same test (18). However, these settings are far away from the typical routines and requirements of a GP practice. Validation data and feasibility studies directly from general practices do not yet exist to our knowledge and are urgently needed.

The aims of this study were twofold: (1) to evaluate the diagnostic accuracy of a SARS-CoV-2 rapid antigen test in primary care and (2) to investigate how well the test is accepted by the practice staff.

\section{Material And Methods}

Study design

This prospective multicenter validation study in general practice was conducted in 40 general practices in Würzburg, a city of nearly 130,000 inhabitants in the federal state of Bavaria, and its surrounding area from December 2020 to June 2021

Study population

Inclusion criteria for patients were based on the guidelines of the Robert Koch Institute (19), the national public health authority in Germany, which, besides others, is continuously monitoring the COVID-19 situation and estimating the risk for the population. We included adult patients with any respiratory symptoms or symptoms suggesting a SARSCoV-2 infection such as hypo- or anosmia and hypo- or ageusia (20). Participating GPs should check patients for eligibility and include them in the study after written consent. Patients should complete a clinical questionnaire asking for age, sex, contact with a SARS-CoV-2 positive person, symptoms (see Tab. 1) and days since symptom onset. 
Table 1

Description of study population

\begin{tabular}{|c|c|c|}
\hline \multirow[b]{2}{*}{ Variables } & \multicolumn{2}{|c|}{ Prevalence, n: 1.450* } \\
\hline & Test pos.; \% & Test neg.; \% \\
\hline Age; $m^{a}$ & 43 & 40 \\
\hline Contact to a person with COVID-19 & 34.1 & 5.1 \\
\hline \multicolumn{3}{|l|}{ Gender } \\
\hline Female & 45.7 & 46.3 \\
\hline Male & 54.3 & 53.7 \\
\hline \multicolumn{3}{|l|}{ Days since symptom onset } \\
\hline Day 1-3 & 66.7 & 73.4 \\
\hline Day 3-7 & 27 & 20.6 \\
\hline Day $>7$ & 6.3 & 6 \\
\hline \multicolumn{3}{|c|}{ *missing values range between 81 and 136} \\
\hline
\end{tabular}

Test procedure

GP took two nasopharyngeal swabs from each patient. The first swab for the point of care test (POCT) and the second swab for PCR testing. According to the manufacturer's protocol of the Roche SARS-CoV-2 Rapid Antigen Test (21), the practice staff read the result of the antigen test after 15-30 minutes. If the test showed a clear positive result earlier than 15 minutes, this positive test result was considered as valid. The second swab for RT-PCR was sent to one of six different cooperating laboratories. All laboratories were certified according to DIN EN ISO 15189:2014 or ISO 9001:2015 $(22,23)$. If one of the two tests (POCT or RT-PCR) was positive, an antibody (Ab) serology was offered on a voluntary basis. The blood sample was taken at the respective GP office earliest ten days after positive test result. The analysis was conducted at the Institute for Hygiene and Microbiology, Würzburg. All test results and the symptom questionnaire were linked by a study ID.

Sample size calculation and statistical analysis

For the evaluation of the antigen test compared to PCR results, a $95 \%$ confidence interval of $+/-5 \%$ for sensitivity and specificity was considered adequate. Assuming a prevalence of $9 \%$, a sensitivity of $78 \%$ and a specificity of $99 \%$, the required sample size was 2930 participants.

Baseline characteristics of patients were analyzed descriptively and were expressed as percent, median with interquartile range (IQR) or mean with standard deviation (SD). To test for differences between groups, Fischer's exact two-tailed tests were performed in case of nominal variables and the Mann-Whitney $U$ test in case of metric and categorical variables. 
To determine the accuracy of the antigen tests, we calculated specificity, sensitivity, positive and negative predictive value (PPV, NPV), with their 95\% confidence intervals (95\% CI). The values were calculated using the RT-PCR result as a reference. We broke down the cycle threshold (Ct-) values as a proxy for the viral load into groups and determined the sensitivity for each group. We split the PCR-positive results into two groups. In one group, PCR and POCT results matched (true positive), in the other the POCT falsely showed a negative result (false negative). The difference between the two groups was plotted as a function of the Ct value to determine the specific difference.

Survey of medical staff

GPs and medical assistant (MA) who were involved in the organization, implementation and execution of the rapid antigen test were asked using a standardized questionnaire about the feasibility and acceptance of the procedure at the end of the study. After eliciting some baseline characteristics, GPs and assistants could rate the organizational and logistical effort of the test procedure as well as consequences for medical treatment on six-point Likert scales, for example ranging from very good to very bad or not useful to very useful. In addition, we encouraged participants to provide free text responses in order to achieve a higher data saturation (for the detailed questionnaire, see attachment). For the conduct of the survey we used the survey software EvaSys (24).

Data analyses were performed using IBM SPSS Statistics (Version 26) software (25).

\section{Results}

Study population

The participating 40 general practices recruited 1,518 patients, on average, 36.3 (SD 27) patients per practice (Fig. 1). After exclusion of 68 patients $(0.04 \%)$, because they did not meet inclusion criteria, the final sample consisted of 1450 patients, $1386(95.6 \%)$ of them partly or fully completed the symptom questionnaire.

Of the included patients, 53\% (730/1450) were female; median age was 40 years (IQR 25) and the median time between symptom onset and consultation was two days (IQR 1.5). Most patients (86\%, 1253/1450) consulted their GP within the first 7 days after symptom onset (Table 1).

Diagnostic accuracy of the rapid antigen test

Of the patients, 9.5\% (138/1450) had a PCR-positive test. Of these, 78.3\% (108/138) also had a positive antigen test result, 30 patients had negative results (considered as false negative result). Seven out of 1312 PCR negative had positive rapid antigen test results (considered false positive), yielding a specificity of $99.5 \%$. In our study population with a SARS-CoV-2 prevalence of $9.5 \%$, the rapid test achieved a PPV of $93.9 \%$ and an NPV of $97.8 \%$ (Table 2).

Table 2 Overview of the results: Comparison of the rapid antigen test and PCR for SARS-CoV-2. 


\begin{tabular}{|c|c|c|c|c|}
\hline \multirow{5}{*}{ Rapid antigen test } & \multirow[b]{3}{*}{ Positiv } & \multicolumn{3}{|c|}{ RT-PCR } \\
\hline & & Positiv & Negativ & Total \\
\hline & & 108 & 7 & 115 \\
\hline & Negativ & 30 & 1305 & 1335 \\
\hline & Total & 138 & 1312 & 1450 \\
\hline & & $\%$ & \multicolumn{2}{|c|}{$(95 \% \mathrm{CI})^{\mathrm{a}}$} \\
\hline Prevalence & & 9.5 & & \\
\hline Specificity & & 99.5 & \multicolumn{2}{|c|}{$(99.0-99.8)$} \\
\hline Sensitivity & & 78.3 & \multicolumn{2}{|c|}{$(70.9-84.6)$} \\
\hline NPV & & 97.8 & \multicolumn{2}{|c|}{$(96.9-98.5)$} \\
\hline PPV & & 93.9 & \multicolumn{2}{|c|}{$(88.6-97.3)$} \\
\hline
\end{tabular}

$\mathrm{a}_{95} \% \mathrm{CI}=95 \%$ confidence intervall.

We investigated the sensitivity in relation to the Ct value. The median Ct-value was 23 (IQR 6.8). The detection rate decreased significantly with increasing (Ct-value i.e. decreasing viral load). For example, the sensitivity for patients with a Ct-value of $>30$ was only $25 \%$ ( $n=12,95 \% \mathrm{Cl}: 7.7 \%-57.2 \%)$ (Fig. 2). We were able show that the Ct values for true positive results were significantly lower than for false negative results ( $p$-value: <0.005). Figure 3 shows the dispersion of the individual test results (true positive, false negative) over the Ct-value scale.

\section{Serological Testing}

The opportunity to determine seroconversion was offered to 145 patients who tested positive, 51 (35.2\%) accepted the offer.

We defined seven cases as "false positive" (Table 2). Of the seven patients, we performed a serology in three cases. Against expectations, all of them showed a clear positive seroconversion. Independently of this study, we received reports from the GPs that two of them underwent further RT-PCR which led to positive results.

Furthermore, we defined 30 cases as "false negative" (Tab.2). Of these, we performed serology in eleven cases, only six cases showed seroconversion with positive Ab-titers. Five cases showed a negative Ab-titer.

Moreover, we performed serology in 37 cases defined as "true positive". Here, 35 cases showed a clearly positive seroconversion.

Acceptance of the test by the practice staff

A total of $40 \mathrm{GPs}$ and $39 \mathrm{MA}$ completed the survey to evaluate the acceptance of the POCT. Almost half of the GPs were female (48.6\%), $46 \%$ of them were between 45 and 54 years old and $61 \%$ worked in a group practice. The assistants were mostly female (92\%), $58 \%$ of them were between 35 and 54 years old and they were predominantly $(62 \%)$ active in a group practice. For more detailed information see Table 1 in the attachment.

Feasibility of the test procedure. 
The participants estimated that pure working time for the execution of a test was, in median, 5 (IQR 4) minutes. Overall, the GPs rated the performance as easy, with an average score of 2.0 on a scale from 1 (very simple) to 6 (very complicated). The MAs perceived the feasibility of the tests in everyday practice as moderate, with an average score of 2.7 ( $n=39$, SD 0.9 ) on a scale of 1 (very good) to 6 (very bad). In the free text section of this question, MAs reported the increased documentation work as a burden.

\section{Perceived benefits of the antigen test.}

GPs and MAs largely agreed that the use of rapid antigen testing could have a positive impact on infection control. On a scale from 1 (strongly agree) to 6 (strongly disagree), GPs rated the statement with 4.5 (SD1.5) and MAs with 4.5 (SD1.4). Nearly all of the GPs (92.1\%) and assistants (94.6\%) agreed that the physician-performed tests were superior to lay test (over-the-counter tests). GPs reported that the antigen test helped them to decide on further interventions in more than half of the tested patients (5.3/10, SD 3.9). Consequences of the antigen test results were, for example, an earlier initiated quarantine, rapid risk assessment and early close-meshed care in case of a positive result (Tab. 3). Almost two thirds $(64.1 \%, n=39)$ of GPs even stated that there were situations in which they would prefer to use rapid antigen tests in the first place. Particularly in situations in which rapid decision-making is necessary, for example, in symptomatic high-risk patients or emergency cases that need to be admitted to the hospital (Tab. 3).

Table 3

Representative citations to the free text sections of the survey. (Original language German)

\section{Situations in which the POCT was a help for further medical treatment}

GP "Rapid statement also in the context of contact tracing as the PCR test often takes a long time. One patient tested positive in a POCT prior to the weekend $\diamond$ close-meshed telephone "monitoring" then still in time hospitalization".

GP "Positive rapid test with corresponding symptoms clearly indicates suspicion of COVID. As a consequence, quarantine measures and notification to health department have already been possible prior to the weekend."

Situations in which the POCT is preferred.

GP "Home visit, decision on hospital admission, high-risk individuals."

GP "If symptoms are severe: high fever, dyspnea, reduced general health, family accumulation of disease, preexisting diseases such as COPD or asthma, or immunosuppressed patients"

GP "Before visiting sick person if PCR test result would take too long."

Note. Original language German

\section{Discussion}

Given the common use of rapid testing in GP practices, there was a need in data on performance at this particular "point of care" and to learn more about whether and how well these tests can be used in practice.

For the Roche SARS-CoV-2 Rapid Antigen Test we used in this study we could show a very high specificity, similar to the manufacturer specifications. The measured sensitivity of $78 \%$ was far lower than the manufacturer's reported sensitivity of $96.25 \%$ (21). The Paul Ehrlich Insitute (PEI - German Federal Institute for Vaccines and Biomedicines) determined an overall sensitivity of $45 \%$ only. The sensitivity for the validation samples with a $\mathrm{Ct}$ value $\leq 25$ was still $88.9 \%$ (26). As shown by the PEI data, we were able to prove that the sensitivity decreased with increasing Ct-values, 
i.e. decreasing viral load. GPs and medical assistants found the POCT easy to implement into everyday practice. Even though its performance is lower than a PCR test, many GPs would use a rapid antigen test especially in situations with an immediate need for action.

Performance of the rapid antigen test

Professionally conducted antigen tests do not require manufacturer-independent validation to be approved for the German market (27). When investigated in independent clinical studies, the sensitivities of the Roche SARS-CoV-2 Rapid Antigen Test in symptomatic patients ranged from less than $63 \%$ to well over $85 \%$ (28-31). The different results can probably be explained by different study characteristics (test site, inclusion criteria, staff training, etc.). Therefore, external manufacturer-independent validation in different clinical settings is needed. This allows diagnostic accuracy and scope of a test result to be interpreted correctly.

Like other studies before, our results show that the test provides reliable results especially at very low Ct-values, i.e. at a high viral load (15). The viral load is dependent on the days since symptom onset. At the beginning of the infection, the viral load is particularly high and decreases over time $(30,32)$. The Ct-value alone is not sufficient to exclude risk of transmission. Infectivity is significantly dependent on SARS-CoV-2 gene copy number as well as genome integrity (33). However, the Ct-value correlates strongly with the viral load and for this reason, it can be a valuable tool for decision making and risk assessment (34). Above a Ct-value of $>30$, virus cultivation is difficult and infectivity seems unlikely (35). We showed that the POCT does not always provide reliable results even at a Ct-value of less than 30 (Fig. 3), a range of diagnostic inaccuracy prevails especially at Ct-values between 20 and 30.

Our results showed a sufficiently high specificity of antigen tests in primary care practices. However, even if positive test results can be interpreted with high probability as true positives, they should be confirmed by a RT-PCR. In contrast, the sensitivity of the test was only moderate and strongly depended on the viral load. Rapid antigen tests cannot provide information about the viral load, and thresholds to rule out contagiousness are difficult to define. Furthermore, the test cannot distinguish between a beginning and a declining infection. If a patient is at the beginning of the infection, the viral load may have increased after a short time and infection of other people cannot be ruled out. A negative test result cannot exclude a transmission-relevant infection with certainty and should not lead to a false sense of security (36).

The high mutation potential of the coronavirus is also a recurring topic of discussion. The mutations mostly affect the S-protein. Rapid tests, on the other hand, usually detect the N-protein. Thus, the tests should still be able to detect infection even in the presence of mutations (37). In the case of since recently dominating the omicron variant, however, initial data show that the sensitivity of the rapid test could be lower (38). Regular re-evaluations seems absolutely necessary to detect diagnostic deficiencies in time.

The PPV and NPV depend on the prevalence, and these parameters are highly dynamic as the pandemic is strongly fluctuating. This should still be kept in mind when interpreting the results.

Feasibility and benefits of the rapid antigen test

The additional test caused only minor logistical challenges for GPs and MA. Its regular implementation in everyday practice should be easily possible. GPs and MA considered a physician-performed test to be superior to lay testing because it is presumed to be more accurately administered. Vice versa, the public also seems to have the highest trust in physician-performed antigen tests. According to a survey in Germany, general practices are the preferred testing location for $33 \%$ of patients, pharmacies for $25 \%$ and testing centers for only $12 \%$ (14). 
GPs considered positive test results helpful as they were perceived as very safe due to their high specificity and appropriate measures such as quarantine and specific medical care could be initiated immediately.

Strengths and weaknesses

Our RT-PCR samples were evaluated at different accredited laboratories. Despite of a consistently high quality standard, the Ct-values are only comparable to a limited extent. However, it can be assumed that the trends (high Ctvalue=low viral load, low Ct-value=high viral load) are still correct. Furthermore, we could not consider the Ct-values of all positive patients because in some cases they were not documented by the practice or provided by the laboratories.

Two different nasopharyngeal swabs were taken consecutively from each patient. Differences in the two sample collections cannot be excluded.

MAs rated the feasibility of the tests worse than the GPs. In some cases, the additional documentation, which was needed for the study, was stated as a reason. In everyday practice without this documentation, the feasibility should achieve better ratings.

To further verify the results of the POCT and especially PCR testing, we offered antibody testing to all positive tested participants. Unfortunately, only one third of the patients took up this voluntary offer. However, although the performed serologies were able to confirm an infection in most of the cases, they also lead to the suggestion that the tests, including the RT-PCR test, do not always provide error-free results. Probably, a second additional PCR test could provide more reliability in future studies.

\section{Conclusions}

A rapid antigen test is a feasible diagnostic tool for the detection of a SARS-CoV-2 infection in general practice. It showed a sensitivity below the manufacturer's specification in the practice but high values for specificity and high PPV and NPV. Given these values, implementation requires a sensitive interpretation of the results in order to derive measures for further treatment. GPs should be aware of the false negative test results as a possible diagnostic gap.

\section{Abbreviations}




\begin{tabular}{ll} 
Ab & Antibody \\
\hline Cl & Confidence interval \\
\hline COVID-19 & Coronavirus disease 2019 \\
\hline Ct & Cycle threshold \\
\hline GP & General practitioner \\
\hline IQR & Interquartile range \\
\hline MA & Medical assistant \\
\hline NPV & Negative predictive value \\
\hline OR & Odds ratio \\
\hline POCT & Point of care test \\
\hline PPV & Positive predictive value \\
\hline (RT-)PCR & (Reverse transcription) polymerase chain reaction \\
\hline SARS-CoV-2 & Severe acute respiratory syndrome coronavirus type 2 \\
\hline SD & Standard deviation \\
\hline
\end{tabular}

\section{Declarations}

Ethics approval and consent to participate

Prior to participation in the study, each patient was informed verbally and by letter about the study procedure. Afterwards, an informed consent was signed from each patient. To assure anonymized documentation, each patient was randomly assigned an individual study ID. Participation in the survey was voluntary and anonymous for the GPS and MAs. The study was reviewed and approved by the ethics committee of the University of Wuerzburg (254/20$\mathrm{sc})$.

Consent for publication

Not applicable

Availability of data and materials

The datasets used and/or analyzed during the current study are available from the corresponding author on reasonable request.

Competing interests

The authors declare that they have no competing interests.

Funding

The study was financed by institutional budget from the Department of General Practice, Wuerzburg and the Free State of Bavaria, Germany, with COVID research funds provided to the University of Wuerzburg (to OK). The Health Department of Wuerzburg supported the study by providing rapid tests. The health department was not involved in the planning or execution of the study. 
Authors' contributions

IG and HS conceptualized the study, with support from OK, TL, JW and WH. IG, HS and JR designed the study materials. JR was responsible for the execution and data collection. OK was the contact person for microbiological issues and responsible for Ab-testing. WH and JR participated in the statistical analyses. JR prepared the first draft of the manuscript. All authors participated in editing the draft and approved the final manuscript.

Acknowledgements

We would like to sincerely express our gratitude to all practices that participated in this study:

Praxis Dr. Artyunyan, Hausarztzentrum Albertshofen, Praxis Dr. Heiduk und Dr. Heßdörfer, Mainschleifenpraxis Volkach, Praxis Dr. Häusner und Söder, Praxis Dr. Legat, Praxis Dr. Mack-Burkhardt, Oberlandpraxis Stadtlauringen, Praxis Dr. Stefan Brugger, Hausärztliche Praxis Rottenbauer, Hausarztpraxis in der Echtergalerie, Praxis Dr. Kluge, Praxis Dr. Gresky, Praxis Dr. Jain, Praxis Dres. Schauber und Hirtl, Praxis Schöneberger-Schanow und Schmitt, Praxis Dres. Kissling und Müller, Praxis Dr. Tschammler, Praxis Dres. Glück und Osiander, Praxis Dres. Geis, Bockholdt und Grebner, Praxis Silke Daci, Praxis Dres. Kuhn, Rosenbauer, Samper-Drösch, Praxis Dres. Sauer und Lohmann, Praxis Dr. Escher, Praxis Dres. Pfeiffer, Praxis Dr. Marouche, Praxis Dr. Vescovi - Dr. Zipperich und Kollegen, Praxis Dr. Becker und Thum, Praxis Dr. Rybol, Praxis Dr. Schenk, Praxis Dr. Rubenbauer, Hausarztzentrum Grafenrheinfeld, Kellermühle Gemeinschaftspraxis, Praxis Dres. Langmann und Schmitt, Praxis Dr. Brack, Praxis Dr. Fuchs, Praxis Konrad Mittenzwei, Praxis Dres. Uebel, Nittka und Kollegen, Praxis Dr. Konz

\section{References}

1. WHO. Laboratory testing for coronavirus disease (COVID-19) in suspected human cases: interim guidance 03/2021.

2. Corman VM, Landt O, Kaiser M, Molenkamp R, Meijer A, Chu DK, et al. Detection of 2019 novel coronavirus (2019-nCoV) by real-time RT-PCR. Euro Surveill. 2020;25(3).

3. Ndwandwe D, Mathebula L, Kamadjeu R, Wiysonge CS. Cochrane corner: rapid point-of-care antigen and molecular-based tests for the diagnosis of COVID-19 infection. Pan Afr Med J. 2020;37(Suppl 1):10.

4. Fomsgaard AS, Rosenstierne MW. An alternative workflow for molecular detection of SARS-CoV-2 - escape from the NA extraction kit-shortage, Copenhagen, Denmark, March 2020. Euro Surveill. 2020;25(14).

5. Péré H, Podglajen I, Wack M, Flamarion E, Mirault T, Goudot G, et al. Nasal Swab Sampling for SARS-CoV-2: a Convenient Alternative in Times of Nasopharyngeal Swab Shortage. J Clin Microbiol. 2020;58(6).

6. Di Muzio F, Barucco M, Guerriero F. Diagnosis and treatment of acute pharyngitis/tonsillitis: a preliminary observational study in General Medicine. Eur Rev Med Pharmacol Sci. 2016;20(23):4950-4.

7. Arbeitsgemeinschaft der Wissenschaftlichen Medizinischen Fachgesellschaften. Therapie entzündlicher Erkrankungen der Gaumenmandeln - Tonsillitis. 2015.

8. Basile K, Kok J, Dwyer DE. Point-of-care diagnostics for respiratory viral infections. Expert Rev Mol Diagn. 2018;18(1):75-83.

9. Dinnes J, Deeks JJ, Adriano A, Berhane S, Davenport C, Dittrich S, et al. Rapid, point-of-care antigen and molecular-based tests for diagnosis of SARS-CoV-2 infection. Cochrane Database Syst Rev. 2020;8(8):Cd013705.

10. Kippen R, O'Sullivan B, Hickson H, Leach M, Wallace G. A national survey of COVID-19 challenges, responses and effects in Australian general practice. Australian Journal for General Practitioners. 2020;49:745-51.

Page $11 / 17$ 
11. Bfarm. Medizinprodukte, Hinweise zur Liste der Antigen-Tests nach §1 Satz 1 Coronavirus-Testverordnung TestV. 03/2021.

12. WIdO - Wissenschaftliches Institut der AOK. Abrechnungsdaten von AOK-Versicherten gemäß § 295 SGB V. 2020.

13. Lee JQ, Loke W, Ng QX. The Role of Family Physicians in a Pandemic: A Blueprint. Healthcare (Basel). 2020;8(3).

14. Deutsches Ärzteblatt. Viele Menschen würden für kostenfreie Schnelltests zum Hausarzt gehen. Deutsches Ärzteblatt. 03/2021.

15. Wagenhäuser I, Knies K, Rauschenberger V, Eisenmann M, McDonogh M, Petri N, et al. Clinical performance evaluation of SARS-CoV-2 rapid antigen testing in point of care usage in comparison to RT-qPCR. EBioMedicine. 2021;69:103455.

16. Kohmer N, Toptan T, Pallas C, Karaca O, Pfeiffer A, Westhaus S, et al. The Comparative Clinical Performance of Four SARS-CoV-2 Rapid Antigen Tests and Their Correlation to Infectivity In Vitro. J Clin Med. 2021;10(2).

17. Cortés Rubio JA, Costa Zamora MP, Canals Aracil M, Pulgar Feio M, Mata Martínez A, Carrasco Munera A. [Evaluation of the diagnostic test for rapid detection of covid-19 antigen (Panbio Covid rapid test) in primary care]. Semergen. 2021;47(8):508-14.

18. Landaas ET, Storm ML, Tollånes MC, Barlinn R, Kran AB, Bragstad K, et al. Diagnostic performance of a SARSCoV-2 rapid antigen test in a large, Norwegian cohort. J Clin Virol. 2021;137:104789.

19. Robert Koch Institut RKI. 2021 [Available from: https://www.rki.de/EN/Home/homepage_node.html.

20. RKI. Testkriterien für die SARS-CoV-2 Diagnostik bei symptomatischen Patienten mit Verdacht auf COVID-19. 2021 [Available from:

https://www.rki.de/DE/Content/InfAZ/N/Neuartiges_Coronavirus/Teststrategie/Testkriterien_Herbst_Winter.html.

21. Roche - SD BIOSENSOR. SARS-CoV-2 Rapid Antigen Test - Packungsbeilage. 2021.

22. Zimmermann U, Steinhorst A. DIN EN ISO 15189. In: Gressner AM, Arndt T, editors. Lexikon der Medizinischen Laboratoriumsdiagnostik. Berlin, Heidelberg: Springer Berlin Heidelberg; 2019. p. 706-.

23. DIN EN ISO 9001, (2015).

24. Electric Paper Evaluationssysteme GmbH. EvaSys V8.0. 2020.

25. IBM Corp.: Armonk N, USA,. IBM SPSS Statistics für Windows, Fassung 26. 2019.

26. Paul Ehrlich Insitut. Comparative evaluation of the sensitivities of SARSCoV-2 antigen rapid tests 2022 [Available from: https://www.pei.de/SharedDocs/Downloads/DE/newsroom/dossiers/evaluierung-sensitivitaetsars-cov-2-antigentests.pdf?_blob=publicationFile\&v=73.

27. Paul-Ehrlich-Insitut - PEl. Mindestkriterien für SARS-CoV-2 Antigentests im Sinne von § 1 Abs. 1 Satz 1 TestVO: Antigenschnelltests. In: PEl, editor. 2021.

28. Schuit E, Veldhuijzen IK, Venekamp RP, van den Bijllaardt W, Pas SD, Lodder EB, et al. Diagnostic accuracy of rapid antigen tests in asymptomatic and presymptomatic close contacts of individuals with confirmed SARSCoV-2 infection: cross sectional study. Bmj. 2021;374:n1676.

29. Möckel M, Corman VM, Stegemann MS, Hofmann J, Stein A, Jones TC, et al. SARS-CoV-2 antigen rapid immunoassay for diagnosis of COVID-19 in the emergency department. Biomarkers. 2021;26(3):213-20.

30. Igloi Z, Velzing J, van Beek J, van de Vijver D, Aron G, Ensing R, et al. Clinical Evaluation of Roche SD Biosensor Rapid Antigen Test for SARS-CoV-2 in Municipal Health Service Testing Site, the Netherlands. Emerg Infect Dis. 2021;27(5):1323-9.

31. Jegerlehner S, Suter-Riniker F, Jent P, Bittel P, Nagler M. Diagnostic accuracy of a SARS-CoV-2 rapid antigen test in real-life clinical settings. Int J Infect Dis. 2021;109:118-22. 
32. Bulilete O, Lorente P, Leiva A, Carandell E, Oliver A, Rojo E, et al. Panbio ${ }^{\text {TM }}$ rapid antigen test for SARS-CoV-2 has acceptable accuracy in symptomatic patients in primary health care. J Infect. 2021;82(3):391-8.

33. Huang CG, Lee KM, Hsiao MJ, Yang SL, Huang PN, Gong YN, et al. Culture-Based Virus Isolation To Evaluate Potential Infectivity of Clinical Specimens Tested for COVID-19. J Clin Microbiol. 2020;58(8).

34. Yu F, Yan L, Wang N, Yang S, Wang L, Tang Y, et al. Quantitative Detection and Viral Load Analysis of SARS-CoV2 in Infected Patients. Clin Infect Dis. 2020;71(15):793-8.

35. Laferl H, Kelani H, Seitz T, Holzer B, Zimpernik I, Steinrigl A, et al. An approach to lifting self-isolation for health care workers with prolonged shedding of SARS-CoV-2 RNA. Infection. 2021;49(1):95-101.

36. RKI. Epidemiologisches Bulletin 17/2021. Robert Koch Institut; 2021.

37. Paul Ehrlich Insitut. SARS-CoV-2-Antigentests für Nachweis der Omikron-Infektion geeignet 2021 [updated 31.12.2021. Available from: https://www.pei.de/DE/newsroom/hp-meldungen/2021/211230-antigentestsomikron-variante.html.

38. U.S. Food and Drug Administration. SARS-CoV-2 Viral Mutations: Impact on COVID-19 Tests 2021 [Available from: https://www.fda.gov/medical-devices/coronavirus-covid-19-and-medical-devices/sars-cov-2-viralmutations-impact-covid-19-tests\#omicronvariantimpact.

\section{Attachment \\ Questionnaire}

See supplementary file 1 (Questionnaire MA) and 2 (Questionnaire GP)

Survey: Baseline characteristics

Attachment table 1: Baseline characteristics of MAs and GPs who followed the survey 


$$
\mathrm{GP}^{\mathrm{a}}, \mathrm{n}=40^{*} \quad \mathrm{MA}^{\mathrm{a}}, \mathrm{n}=39 \text { * }
$$
n $(\%)$
n $\quad(\%)$

\begin{tabular}{|c|c|c|c|c|}
\hline Sex & & & & \\
\hline Male & 19 & $(51.4)$ & 3 & $(7.9)$ \\
\hline Female & 18 & $(48.6)$ & 35 & $(92.1)$ \\
\hline \multicolumn{5}{|l|}{ Age } \\
\hline$<35$ & 2 & $(5.4)$ & 10 & $(27.8)$ \\
\hline $35-54$ & 24 & $(64.9)$ & 21 & $(58.3)$ \\
\hline $55-74$ & 11 & $(29.7)$ & 5 & $(13.9)$ \\
\hline \multicolumn{5}{|c|}{ Work experience in years } \\
\hline$<5$ & 6 & $(16.7)$ & 10 & $(27)$ \\
\hline $5-9$ & 4 & $(11.1)$ & 3 & $(8.1)$ \\
\hline 10-19 & 11 & $(30.6)$ & 12 & $(32.4)$ \\
\hline$>20$ & 15 & $(41.7)$ & 12 & $(32.4)$ \\
\hline
\end{tabular}

Type of medical practice

\begin{tabular}{lllll}
\hline Community practice & 22 & $(61.1)$ & 23 & $(62.2)$ \\
\hline Single practice & 14 & $(38.9)$ & 14 & $(37.8)$ \\
\hline
\end{tabular}

Location of the practice

\begin{tabular}{lcccc}
\hline $\begin{array}{l}\text { Rural } \\
(<5.000 \text { Inhabitants) }\end{array}$ & 13 & $(36.1)$ & 11 & $(29.7)$ \\
\hline $\begin{array}{l}\text { Small town } \\
(5.000-20.000 \text { Inhabitants) }\end{array}$ & 6 & $(16.7)$ & 7 & $(18.9)$ \\
\hline $\begin{array}{l}\text { Medium size city } \\
(20.000-100.000 \text { Inhabitants) }\end{array}$ & 4 & $(11.1)$ & 4 & $(10.8)$ \\
\hline $\begin{array}{l}\text { Large city } \\
(>100.000 \text { Inhabitants })\end{array}$ & 13 & $(36.1)$ & 15 & $(40.5)$ \\
\hline
\end{tabular}

*missing values range between 1 and 4

${ }^{\mathrm{a}} \mathrm{GP}=$ general practitioner, $\mathrm{MA}=$ medical assistant 
Figures

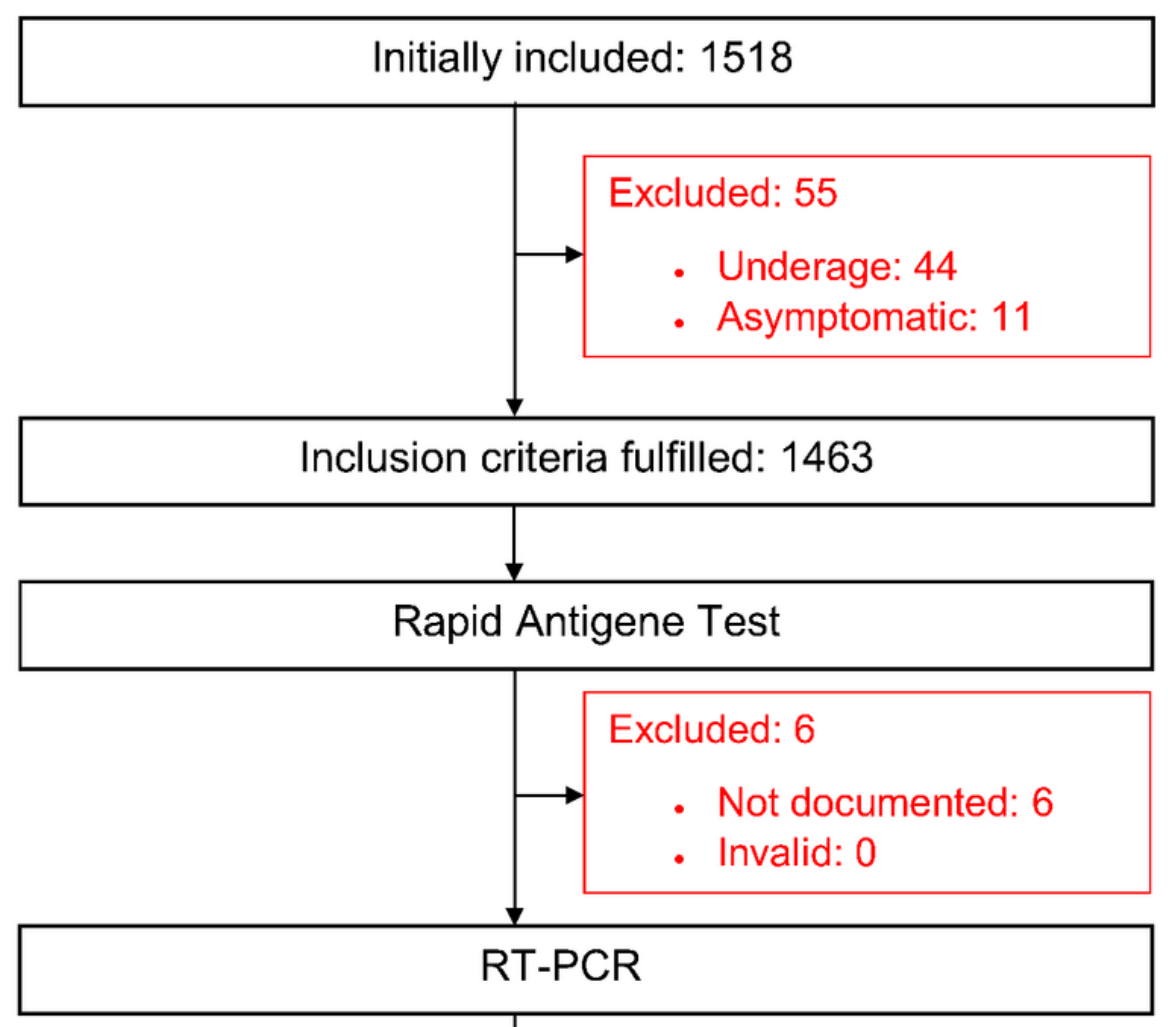

\section{Excluded: 7}

- Not documented: 6

- Invalid: 1

Analyzable datasets with both test results: 1450

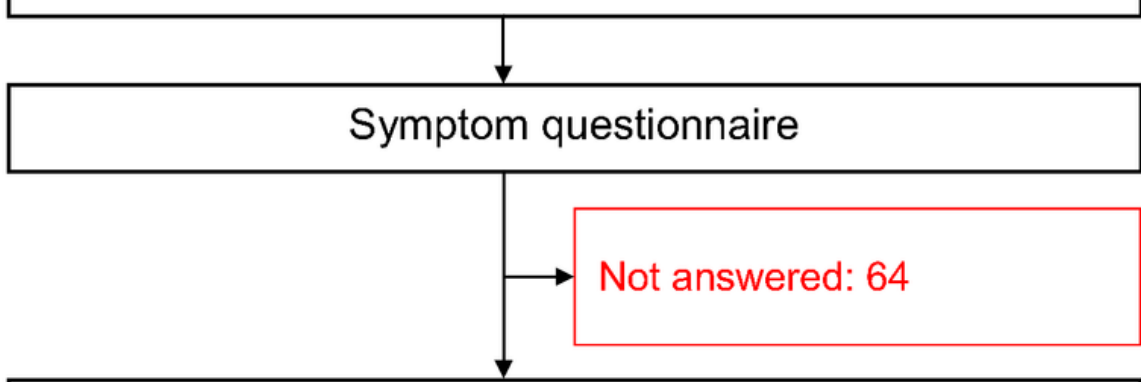

Symptom questionnaires included in analysis: 1386

Figure 1

Flow of study participants: Initially included participants with reasons for exclusion 


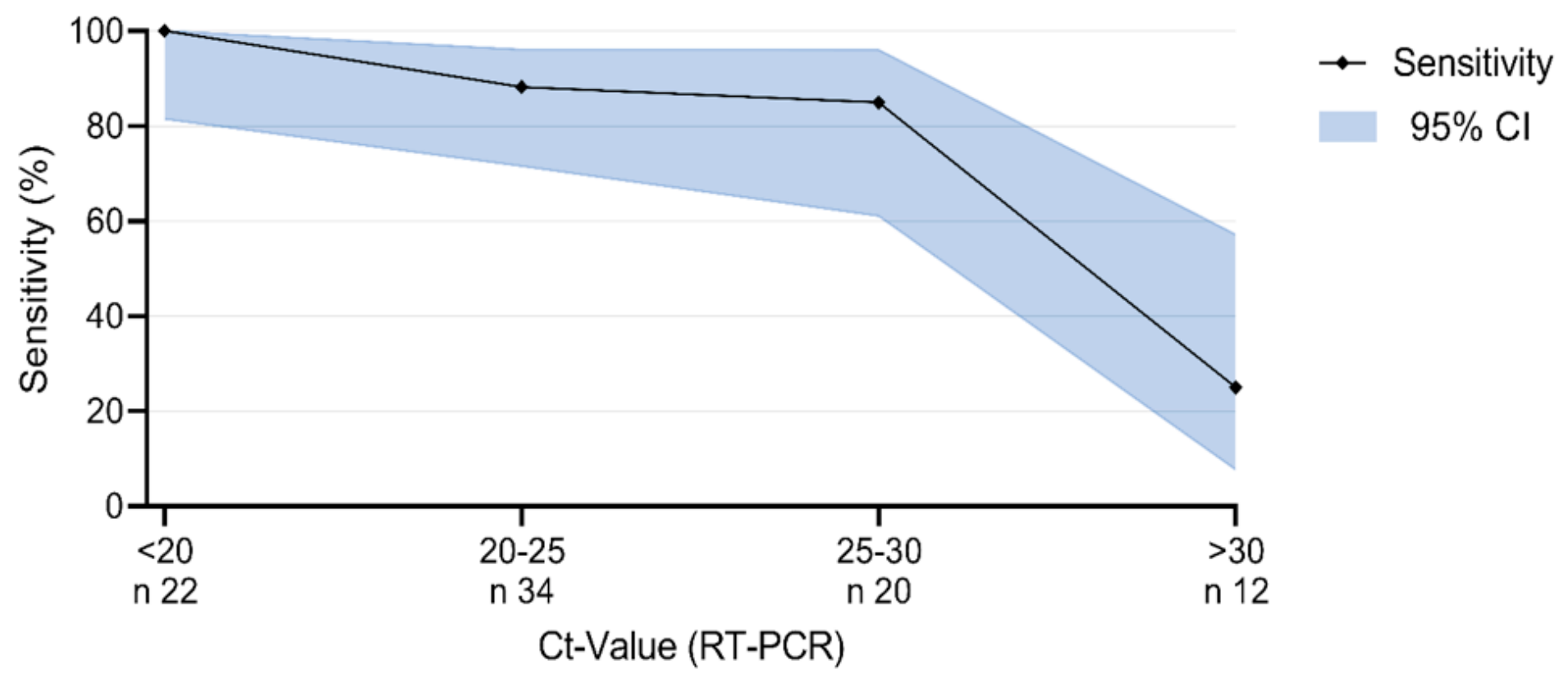

\section{Figure 2}

Sensitivity of the rapid antigen test according to CT-values $(n=88)$.

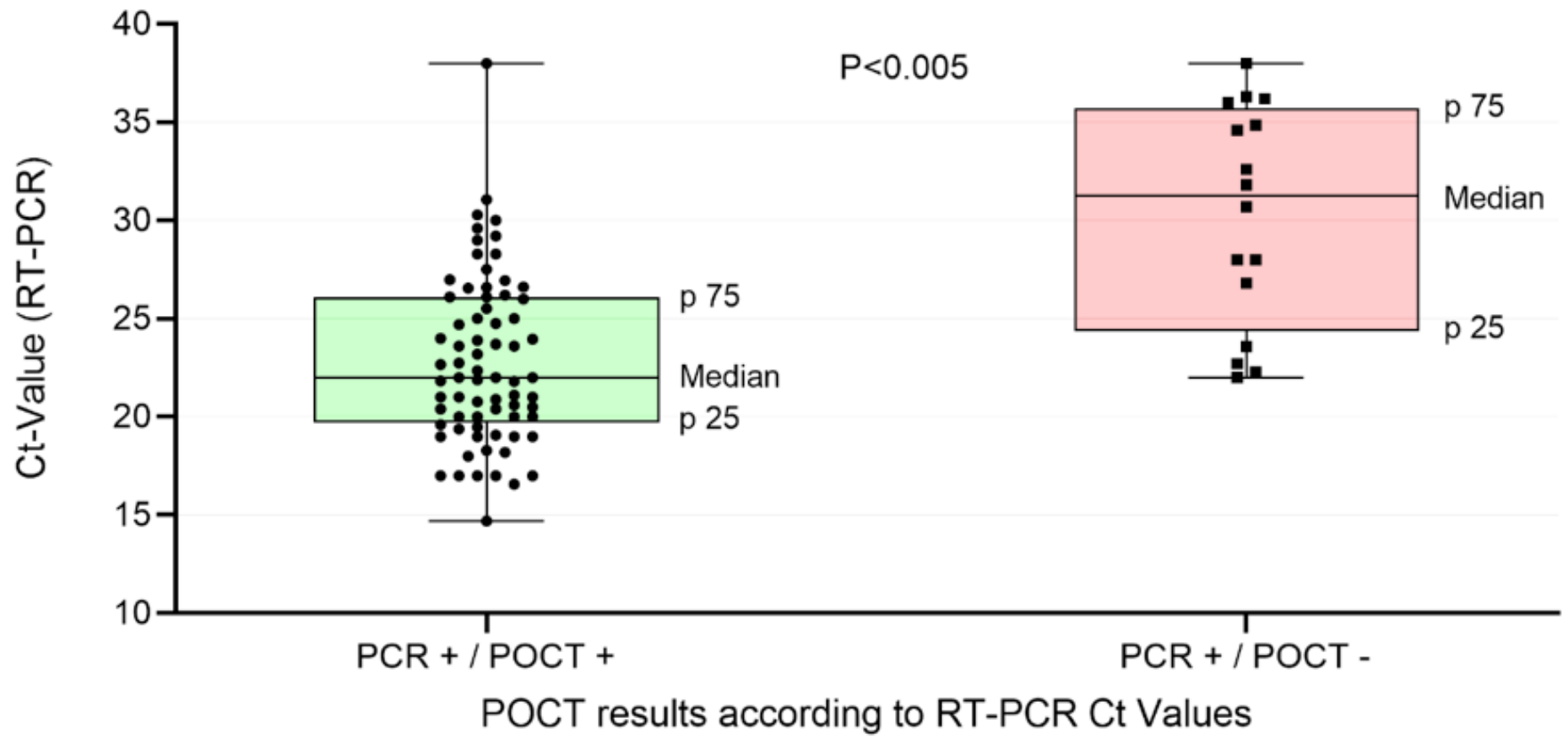

Figure 3

Viral concentration (Ct-value) of true positive rapid antigen tests $(n=72)$ versus false negative tests $(n=16)$.

\section{Supplementary Files}


This is a list of supplementary files associated with this preprint. Click to download.

- QuestionnaireGP.pdf

- QuestionnaireMA.pdf 